\title{
Review of oral cholera vaccines: efficacy in young children
}

\author{
This article was published in the following Dove Press journal: \\ Infection and Drug Resistance \\ I3 September 201 I \\ Number of times this article has been viewed
}

\section{Cristina Masuet Aumatell' \\ JM Ramon Torrell' \\ Jane N Zuckerman² \\ 'International Health Centre, Preventive Medicine Department, Bellvitge Hospital, L'Hospitalet de Llobregat, Barcelona, Spain; ${ }^{2}$ World Health Organization Collaborating Centre for Reference, Research and Training in Travel Medicine, University College London Medical School, London, UK}

Correspondence: Cristina Masuet Aumatell Bellvitge Hospital, International Health Centre, Preventive Medicine Department, Antiga escola d'enfermeria, $4^{a}$ planta, Feixa Llarga s/n, L'Hospitalet de Llobregat, Barcelona 08907, Spain

Tel +34 932607557

Fax +34932607849

Email cmasuet@bellvitgehospital.cat
Background: Young children are one of the most vulnerable groups who may be infected with cholera. The following literature review of the efficacy of the currently available cholera vaccines provides a clear evidence base for the clinical administration of cholera vaccine, particularly in an epidemic situation.

Aim: To assess the efficacy of oral cholera vaccines in preventing cases of cholera in young children.

Methods: A systematic literature review was undertaken for the period 1983 to 2011 using PubMed and the search terms "oral cholera vaccines," "children," and "efficacy," limited to "clinical trials" and "human studies".

Results: Oral cholera vaccine provides an acceptable level of protection in young children, with the level of protection being greater at 12 or 24 months following immunization.

Conclusions: Children exposed to a potential risk of cholera are recommended to be vaccinated with an oral cholera vaccine, irrespective of whether its constituents include the B subunit.

Keywords: efficacy, oral cholera vaccine, children

\section{Introduction}

Cholera epidemiology worldwide, young children $(<5$ years)

Cholera is one of the most severe infectious diseases experienced by children in resource-poor regions of the world, causing a rapidly dehydrating diarrheal disease of mild-to-moderate severity in approximately $90 \%$ of cases, with a case-fatality rate of less than 5\%. ${ }^{1}$ In endemic areas (Africa, Asia, South America, and Central America), infection with cholera results in 3-5 million cases and 100,000-130,000 deaths per year. ${ }^{2}$ Young children, aged less than 5 years, are one of the most vulnerable groups, with an annual incidence rate of cholera 2-4 times higher ${ }^{3}$ than those found in the overall population.

\section{Types of cholera vaccines}

In the early part of the 20th century the injectable killed whole cell vaccination was a required vaccine to enable entry into some countries. Nevertheless this vaccine has not been recommended for many years due to the limited protection and efficacy it afforded, the known side effects and the need for frequent boosters and importantly, the concomitant improvements in public health. ${ }^{4}$

In 1997 a variant whole cell vaccine (vWC) was licensed $\left(\right.$ ORCVAX $\left.^{\circledR}\right)$ in Vietnam for administration to adults and children aged 1 year of age and over. In 2009, this 
vaccine was modified ( ORCVAX $^{\circledR}$ ) to a bivalent vaccine with the high toxin-producing strain being replaced by two Vibrio cholerae strains and including a double quantity of lipopolysaccharide antigen and so replaced the vWC which is no longer available.

Two oral cholera vaccine formulations are currently licensed and available in certain countries: a whole cell plus recombinant B subunit vaccine (WC-rBS); and the bivalent whole cell vaccine (BivWC). ${ }^{5}$ Both vaccines are administered according to a schedule which consists of two doses administered 2 weeks apart which provides protection for 2 years.

The WC-rBS (Dukoral ${ }^{\circledR}$ ) is a monovalent vaccine composed of three strains (classical and El Tor, Inaba and Ogawa) of heat and formalin-killed $V$. cholerae $\mathrm{O} 1$ with an additional purified cholera B subunit. Originally the B subunit of cholera toxin was produced chemically as part of the development of the whole cell plus B subunit vaccine (WC-BS), but currently the vaccine is produced using recombinant technology. The immune response of WC-BS and WC-rBS is practically identical, resulting in the WC-BS cholera vaccine no longer being available. ${ }^{1}$ The WC-rBS has been licensed for use in adults and children aged 2 to 65 years in more than 60 countries and this vaccine is the only one that may provide limited protection against enterotoxigenic Escherichia coli ${ }^{2}$ in travelers. ${ }^{6}$

The BivWC $\left(\right.$ Shanchol $\left.^{\circledR}\right)$ is a bivalent vaccine composed of three strains (classical and El Tor, Inaba and Ogawa) of heat and formalin-killed $V$. cholerae $\mathrm{O} 1$ with an additional strain of formalin-killed $V$. cholerae O139. It is licensed for use in India for adults and children aged 1 year and over.

The vWC and BivWC vaccines do not contain the bacterial toxin $B$ subunit and neither requires a buffer for administration, so making the vaccine less expensive and easier to store as less storage space is required but these vaccines do not provide any protection against enterotoxigenic $E$. coli as one cause of travelers' diarrhea.

A recent meta-analysis ${ }^{7}$ evaluating oral whole cell cholera vaccines but without including age cohorts, demonstrated an efficacy of 52\% (95\% confidence interval [CI]: $35 \%-65 \%)$ in the first year and 61\% (95\% CI: 50\%-70\%) within the second year of follow-up independent of the type of vaccine administered. As young children are one of the most vulnerable groups, who may become seriously ill when exposed to infection with cholera, we completed a literature review of the efficacy of cholera vaccines in this high-risk population.

\section{Material and methods}

A systematic review of the literature was undertaken for the period 1983 to 2011 using PubMed and the search terms "oral cholera vaccines," "children," and "efficacy," but 2011 and limited to "clinical trials" and "human studies". The review included a prescreening of the manuscripts by title and content of the abstracts and these were then assessed in terms of eligibility by reading the full text. Only those studies that described the efficacy of oral whole cell cholera vaccines in children and gave information about the clinical outcome (cases of cholera) and reported the raw data stratified by age ( $\leq 5$ years, $>5$ years old) were included in the review. The review was performed on the efficacy of whole cell cholera vaccines in indigenous children.

The design and possible limitations of the studies were assessed using the criteria applied to randomized control trials (CONSORT guidelines). The main possible limitations considered included any bias because of information, selection, or confounding, which may have led to the over- or underestimation of vaccine efficacy.

Vaccine efficacy was calculated using 95\% CI using the following formula: $(1-$ relative risk $) \times 100 .{ }^{8}$ The relative risk was expressed as incidence of cholera in vaccinees as compared to incidence of cholera in the placebo group.

\section{Results}

The first search resulted in the identification of 38 studies from more than 100 papers and no further papers were included following a comprehensive screening of the references. Several papers ${ }^{9-15}$ which described only the results of interim analyses rather than a full review of a study were excluded from this review. ${ }^{16}$ Similarly, twelve papers which focused on immunogenicity and safety without providing data regarding efficacy were not included. ${ }^{17-26}$ Consequently, four clinical trials involving oral whole cell cholera vaccines were reviewed as follows: WC-BS, ${ }^{27} \mathrm{WC}-\mathrm{rBS},{ }^{28} \mathrm{vWC},{ }^{29}$ and BivWC. ${ }^{30}$ In order to review the optimal evidence base, this review paper was only based on clinical trial data and observational studies were not included.

The study population consisted of indigenous populations of children residing in endemic countries and who were at risk of infection with cholera. Travelers as an at risk group were not considered as part of this review because clinical trials have not been completed in this particular cohort. The four studies included approximately 278,000 people, with approximately 96,000 children aged 1-15 years living throughout Asia and South America (Table 1). Each trial included at least 10,000 children living in a non-outbreak area, with the exception of one study by Trach et al. ${ }^{29}$ These trials provided results for each age group, type of oral cholera vaccine administered as well as for each year of follow-up, although different strata were applied for each clinical trial. 


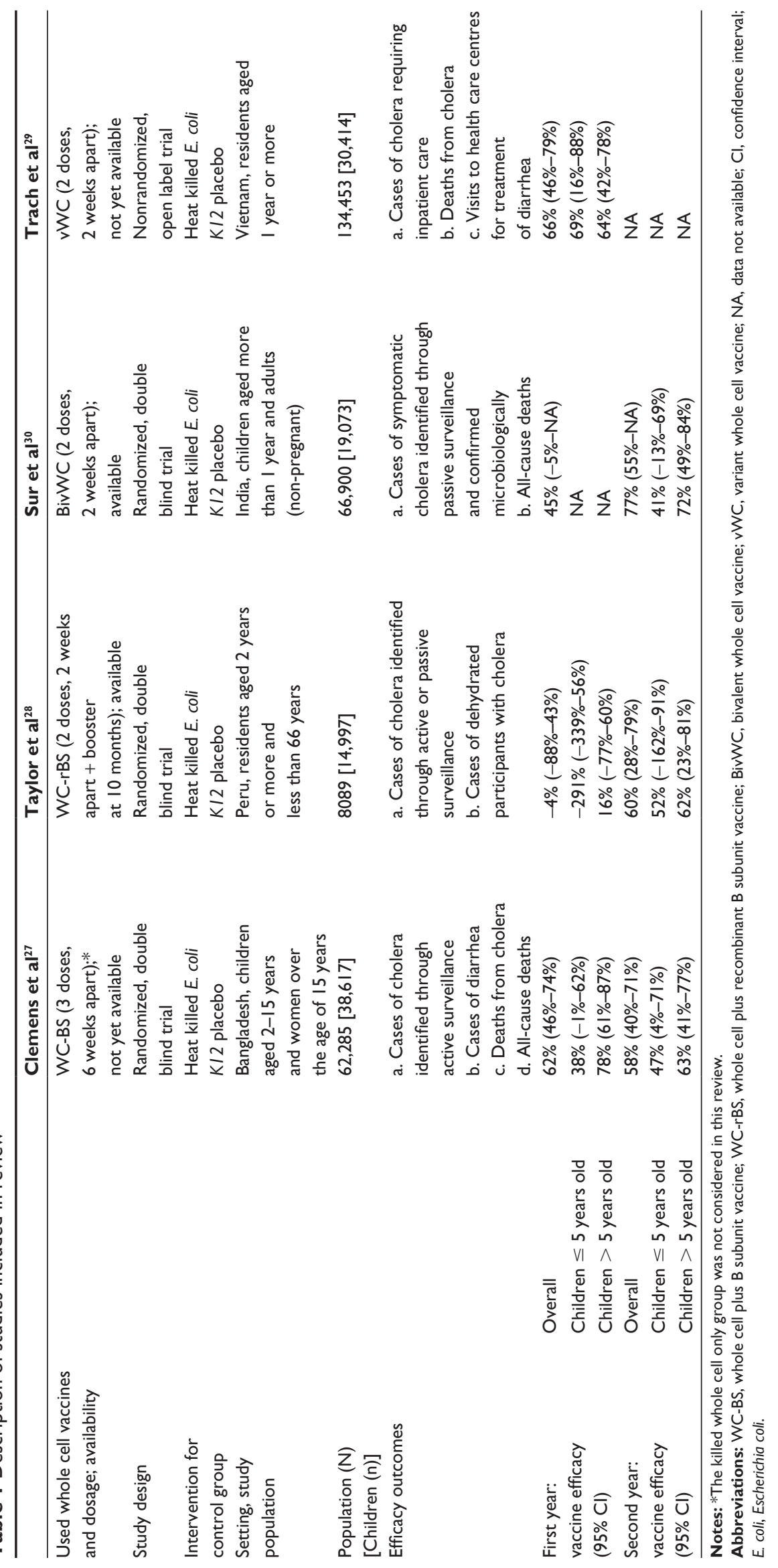


In 1985 , Clemens et $\mathrm{al}^{27}$ conducted a randomized double-blind controlled trial including follow-up over a period of 3 years to assess the protective efficacy of three doses of WC-BS or killed whole cell only oral cholera vaccines administered at 6 -week intervals against placebo (E. coli $\mathrm{K} 12$ ) among 62,285 children and women residing in rural Bangladesh. Only 20,705 people received the WC-BS vaccine and $61.8 \%(n=12,708)$ were children aged 2-15 years. Efficacy was defined through different outcomes based on cases of cholera (symptomatic and asymptomatic), deaths of all causes, and cholera deaths in vaccinees who received WC-BS as compared with the placebo group. The data were stratified according to age: $2-5$ years of age or older than 5 years; and follow-up was undertaken after the at first, second, and third year.

From 1993 to 1995 , Taylor et $\mathrm{al}^{28}$ conducted a randomized double-blind controlled trial with a follow-up of 2 years to assess the protective efficacy of two doses of WC-rBS administered at 2-week intervals followed by a booster 10 months later as compared against placebo (E. coli K12) among 14,997 children and adults up to 65 years old residing in marginal neighborhoods in Lima, Peru. Only 7594 people received the WC-BS vaccine of which $53.9 \%(n=4096)$ were children aged 2-15 years old. The efficacy of the vaccine was defined by different outcomes based on cases of cholera and level of dehydration according to the World Health Organization definitions. These outcomes were identified through active household or passive surveillance, the latter being completed at health centers. The data were stratified by age: $2-5$ years of age or older than 5 years; and follow-up was undertaken after the first and second year.

In 1992, Trach et al ${ }^{29}$ conducted a nonrandomized open-label trial during an outbreak of El Tor cholera with a follow-up of 1 year to assess the protective efficacy of two doses of vWC administered at 2-week intervals as compared with placebo (E. coli $\mathrm{K} 12$ ) among 134,453 residents aged 1 year or more in urban Vietnam (Hue). Half of the population $(n=67,395)$ received the $\mathrm{vWC}$ vaccine and $22.6 \%(\mathrm{n}=15,253)$ were children aged $2-10$ years old. The efficacy was defined by different outcomes based on cases of cholera or diarrhea requiring inpatient care, or deaths from cholera. The data were stratified using the following age groups: $1-5$ years, $6-10$ years, $11-20$ years, $21-40$ years, and older than 40 years; no results were reported regarding the follow-up period.

In 2006, Sur et al ${ }^{30}$ conducted a cluster randomized double-blind controlled trial with a follow-up of 2 years to assess the protective efficacy of two doses of bivalent vaccine (BivWC) administered at 2-week intervals as compared with placebo (E. coli K12) among 66,900 residents aged 1 year or more in urban India (Kolkata). Only 31,932 people received BivWC of which $28.5 \%(n=9,105)$ were children aged 1-14.9 years old. The efficacy was defined by different outcomes based on cases of cholera (first symptomatic cholera episode confirmed by fecal excretion of $V$. cholerae O1 during a nonbloody diarrheal episode) and deaths of all causes in vaccinees as compared with the placebo group. The data were stratified by age group: $1-4.9$ years, $5-14.9$ years, and 15 years or older. The data obtained with reference to the second year of follow-up were only stratified by the age of the population only and results regarding the first year of follow-up were unpublished and unavailable.

\section{Assessment of vaccine efficacy}

Appreciating the heterogeneity of the study methodologies applied and the different oral cholera vaccines administered, the majority of these studies showed an overall protection up to 2 years following vaccination, ranging from $45 \%$ to $77 \%$ in the overall population and between $38 \%$ and $52 \%$ in young children.

In the first year of follow-up, vaccine efficacy ranged from $-4 \%$ to $66 \%$, with the Taylor study confirming the rarity of cholera infection events globally. With respect to the age of the population, this review shows consistently lowered efficacy of the vaccine in children, with a decrease of $5 \%$ to $40 \%$ in young children ( $\leq 5$ years old) compared with the overall population ( $>5$ year old).

During the second year of follow-up, the efficacy of oral cholera vaccine ranged from $58 \%$ to $77 \%$ with a slight increase after the first year of follow-up. With regard to the age of population, a lower efficacy of oral cholera vaccine was evident, with a decrease of $10 \%$ to $31 \%$ in young children ( $\leq 5$ years old) as compared with the overall population $(>5$ year old). An increase in efficacy was evident a year after administration of the vaccine.

The whole cell plus B subunits cholera vaccines (WC-BS and WC-rBS) appear to demonstrate less efficacy during the first year following administration of the vaccine in young children, but higher efficacy in the second year as compared with either the BivWC or vWC cholera vaccines. Further studies are required to confirm this finding, which may be due to imbalances in the $\mathrm{O} 1$ lipopolysaccharide antigen content in the both vaccines.

No other efficacy outcomes could be reviewed due to the absence of stratified data by age groups in each study.

In conclusion, the overall efficacy of oral cholera vaccines is quite acceptable, even though a lower protective effect is 
systematically observed in young children ( $\leq 5$ years old) than as compared with the overall population.

\section{Discussion}

Individual studies have showed that the overall efficacy of oral whole cell vaccines against cholera is slightly higher at 24 months than at 12 months following administration. ${ }^{28}$ Nevertheless the efficacy of an oral cholera vaccine in vulnerable populations remains inadequate and further analyses (meta-analysis) are recommended to understand this.

A herd immunity effect has been described in children in Bangladesh who were too young to be vaccinated with the WC-BS cholera vaccine; vaccinating only $50 \%$ of the population could result in a $93 \%$ reduction in the incidence of cholera in the whole population. ${ }^{31-33}$ It is postulated that any of the available oral cholera vaccines could provide a herd immunity effect for at-risk populations.

One of the problems with the review of these studies was the inclusion of possible biases as although this review includes data analyzed systematically by age groups, a meta-analysis for each age group was not available for inclusion or therefore for review..$^{29,30}$

Other limitations in this review includes the fact that some of the excluded studies were based on immunogenicity or safety data but none of them published data on efficacy by age group.

Furthermore, consideration of the different types of oral whole cell vaccines studied as well as different study settings and vague or different case definitions, could explain the heterogeneity of the available results, which could have led to either over- or underestimation of vaccine efficacy.

\section{Conclusion}

The whole cell oral cholera vaccine provides an acceptable level of efficacy in young children, which is estimated to be lower than in the overall population following the first and second year of administration of the vaccine. A mathematical modeling approach is highly recommended in order to determine the efficacy of oral cholera vaccine before more specific conclusions can be drawn on oral cholera vaccine efficacy in this specific group. Nevertheless, more studies are required to develop this model and to evaluate the effect of herd immunity as a plausible explanation of these findings.

It could also be hypothesized that oral cholera vaccine might decrease the incidence of cholera in children as well as in the overall population. Such a hypothesis requires further blinded and randomized studies in children who would receive oral whole cell vaccines as compared with a non-indigenous populations.

\section{Disclosure}

JN Zuckerman has been a consultant by several manufacturers of vaccines and anti-malarial prophylaxis and has received unrestricted educational grants from GlaxoSmithKline, Novartis, Sanofi Pasteur, SBL Vaccines, and Pfizer, for attending conferences, running educational programmes, and undertaking clinical trials. No other potential conflicts of interest have been reported. C Masuet Aumatell and JM Ramon Torrell have no conflicts of interest to declare.

\section{References}

1. World Health Organization. Cholera vaccines: WHO position paper Wkly Epidemiol Rec. 2010;85(13):117-128.

2. Zuckerman JN, Rombo L, Fisch A. The true burden and risk of cholera: implications for prevention and control. Lancet Infect Dis. 2007;7(8):521-530.

3. Deen JL, von Seidlein L, Sur D, et al. The high burden of cholera in children: comparison of incidence from endemic areas in Asia and Africa. PLoS Negl Trop Dis. 2008;2(2):e173.

4. Tacket CO, Sack DA. Cholera vaccines. In: Plotkin SA, Orenstein WA, Offit PA, editors. Vaccines. 5th ed. Philadelphia, PA: Saunders Elsevier; 2008:127-138.

5. Hill DR, Ford L, Lalloo DG. Oral cholera vaccines: use in clinical practice. Lancet Infect Dis. 2006;6(6):361-373.

6. Torrell JMR, Aumatell CM, Ramos SM, Mestre LG, Salas CM. Reduction in travellers' diarrhoea by WC/rBS oral cholera vaccine in young, high-risk travellers. Vaccine. 2009;27(30):4074-4077.

7. Sinclair D, Abba K, Zaman K, Qadri F, Graves PM. Oral vaccines for preventing cholera [review]. Cochrane Database Syst Rev. 2011;3:CD008603.

8. Orenstein WA, Bernier RH, Dondero TJ, et al. Field evaluation of vaccine efficacy. Bull World Health Organ. 1985;63(6):1055-1068.

9. Clemens JD, Harris JR, Sack DA, et al. Field trial of oral cholera vaccines in Bangladesh. Southeast Asian J Trop Med Public Health. 1988;19(3):417-422.

10. Clemens JD, Sack DA, Chakraborty J, et al. Field trial of oral cholera vaccines in Bangladesh: evaluation of anti-bacterial and anti-toxic breast-milk immunity in response to ingestion of the vaccines. Vaccine. 1990;8(5):469-472.

11. Clemens JD, Sack DA, Harris JR, et al. ABO blood groups and cholera: new observations on specificity of risk and modifications of vaccine efficacy. J Infect Dis. 1989;159(4):770-773.

12. Clemens JD, Sack DA, Harris JR, et al. Field trial of oral cholera vaccines in Bangladesh. Lancet. 1986;2(8499):124-127.

13. Clemens JD, Sack DA, Harris JR, et al. Impact of subunit killed wholecell and killed whole-cell-only oral vaccines against cholera upon treated diarrhoeal illness and mortality in an area endemic for cholera. Lancet. 1988;1(8599):1375-1379.

14. Clemens JD, Sack DA, Harris JR, et al. Field trial of oral cholera vaccines in Bangladesh: results from three-year follow-up. Lancet. 1990;335(8684):270-273.

15. van Loon FP, Clemens JD, Chakraborty J, et al. Field trial of inactivated oral vaccines in Bangladesh: results from 5 years of follow-up. Vaccine. 1996;14(2):162-166.

16. Begue RE, Castellares G, Ruiz R, et al. Community-based assessment of safety and immunogenicity of the whole cell plus recombinant b subunit (WC/rBS) oral cholera vaccine in Peru. Vaccine. 1995;13(7):691-694. 
17. Chen Q, Yu S, Wang Y. Community trial for safety and immunogenicity of oral-administered lyophilized rBS-WC cholera vaccine. Zhonghua Yu Fang Yi Xue Za Zhi. 1996;30(6):330-333. Chinese.

18. Concha A, Giraldo A, Castañeda E, et al. Safety and immunogenicity of oral killed whole cell recombinant B subunit cholera vaccine in Barranquilla, Colombia. Bull Pan Am Health Organ. 1995;29(4):312-321.

19. Hallander HO, Paniagua M, Espinoza F, et al. Calibrated serological techniques demonstrate significant different serum response rates to an oral killed cholera vaccine between Swedish and Nicaraguan children. Vaccine. 2002;21(1-2):138-145.

20. Kanungo S, Paisley A, Lopez AL, et al. Immune responses following one and two doses of the reformulated, bivalent, killed, whole-cell, oral cholera vaccine among adults and children in Kolkata, India: a randomized, placebo-controlled trial. Vaccine. 2009;27(49):6887-6893.

21. Lagos R, Avendaño A, Prado V, et al. Attenuated live cholera vaccine strain CVD 103-HgR elicits significantly higher serum vibriocidal antibody titers in persons of blood group O. Infect Immun. 1995;63(2):707-709.

22. Mahalanabis D, Lopez AL, Sur D, et al. A randomized, placebocontrolled trial of the bivalent killed, whole-cell, oral cholera vaccine in adults and children in a cholera endemic area in Kolkata, India. PLoS One. 2008;3(6):e2323.

23. Qadri F, Chowdhury MI, Faruque SM, et al. Peru-15, a live attenuated oral cholera vaccine, is safe and immunogenic in Bangladeshi toddlers and infants. Vaccine. 2007;25(2):231-238.

24. Richie EE, Punjabi NH, Sidharta YY, et al. Efficacy trial of single dose live oral vaccine CVD 103-HgR in North Jakarta, Indonesia, a cholera endemic area. Vaccine. 2000;18(22):2399-2410.
25. Simanjuntak CH, O'Hanley P, Punjabi NH, et al. Safety, immunogenicity, and transmissibility of single-dose live oral cholera vaccine strain CVD 103-HgR in 24- to 59-month-old Indonesian children. J Infect Dis. 1993;168(5):1169-1176.

26. Suharyono, Simanjuntak C, Witham N, et al. Safety and immunogenicity of single-dose live oral cholera vaccine CVD 103-HgR in 5-9-year-old Indonesian children. Lancet. 1992;340(8821):689-694.

27. Clemens JD, Harris JR, Sack DA, et al. Field trial of oral cholera vaccine in Bangladesh: results from one year of follow-up. J Infect Dis. 1988;158(1):60-69.

28. Taylor DN, Cardenas V, Sanchez JL, et al. Two-year study of the protective efficacy of the oral whole cell plus recombinant B subunit cholera vaccine in Peru. J Infect Dis. 2000;181(5):1667-1673.

29. Trach DD, Clemens JD, Ke NT, et al. Field trial of a locally produced, killed, oral cholera vaccine in Vietnam. Lancet. 1997;349(9047):231-235.

30. Sur D, Lopez AL, Kanungo S, et al. Efficacy and safety of a modified killed-whole-cell oral cholera vaccine in India: an interim analysis of a cluster-randomised, double-blind, placebo-controlled trial. Lancet. 2009;374(9702):1694-1702.

31. Levine MM. Immunogenicity and efficacy of oral vaccines in developing countries: lessons from a live cholera vaccine. BMC Biol. 2010;8:129-138.

32. Sack DA. Herd protection and herd amplification in cholera. J Health Popul Nutr. 2006;24(1):1-5.

33. Ali M, Emch M, von Seidlein L, et al. Herd immunity conferred by killed oral cholera vaccines in Bangladesh: a reanalysis. Lancet. 2005;366(9479):44-49.
Infection and Drug Resistance

\section{Publish your work in this journal}

Infection and Drug Resistance is an international, peer-reviewed openaccess journal that focuses on the optimal treatment of infection (bacterial, fungal and viral) and the development and institution of preventive strategies to minimize the development and spread of resistance. The journal is specifically concerned with the epidemiology of antibiotic

\section{Dovepress}

resistance and the mechanisms of resistance development and diffusion in both hospitals and the community. The manuscript management system is completely online and includes a very quick and fair peerreview system, which is all easy to use. Visit http://www.dovepress.com/ testimonials.php to read real quotes from published authors. 